\title{
L'association
}

\section{dithiocarbamate de pipérazine-arséniate de plomb dans la lutte contre divers helminthes (cestodes et nématodes) du mouton}

par $M . G R A B E R$

La plupart des moutons de la zone sahélienne du Tchad hébergent ainsi qu'il a été dit dans une précédente note (8) un nombre fort important d'helminthes dont l'action, pour n'être pas primordiale dans la pathologie du mouton de ces régions, n'en est pas moins fort importante. $D^{\prime}$ autres facteurs jouent un rôle encore mal connu, mais certainement non négligeable, notamment entre autres, pour ne citer que les plus visibles, le manque d'eau, la disparition ou la raréfaction du pâturage à certaines époques, l'absence ou le petit nombre d'oligo-éléments, etc... C'est lorsque l'équilibre précaire parasite-milieu se trouve rompu sous linfluence des causes extérieures dont il vient d'être question - cette rupture ayant lieu sous ces climats de mai à octobre - que les helminthes, assez bien tolérés en temps normal, provoquent des pertes sensibles variables selon les zones d'élevage. L'état du troupeau reste dans l'ensemble médiocre avec un fort pourcentage de moutons de boucherie dont la qualité laisse fort à désirer. De plus le nombre d'animaux recensés chaque année aurait plutôt tendance à décroître.

Parmi les parasites en cause, les Nématodes et les Cestodes sont les plus largement représentés, les Trématodes (Fasciola-Schistosomes et Paramphistomes) ne se rencontrent en abondance que dans certaines régions assez peu étendues et bien localisées. Nous les laisserons pour l'instant de côté.

Les Cestodes appartiennent à la famille des Anoplocéphalinae (Moniezia expansa et Moniezia benedeni, Stilesia globipunctata, Avitellina centripunctata) et les Nématodes à des groupes divers, les plus nombreux se rattachant aux espèces suivantes : Oesophagostomum columbianum et Haemonchus contortus.
Cestodes et Nématodes se trouvent très souvent associés ainsi qu'en témoigne le tableau suivant portant sur 180 moutons pris au hasard :

Cestodes seuls :

- Moniezia et Stilesia .......

1 animal

- Moniezia et Avitellina ......

- Moniezia, Stilesia et Avitellina

- Stilesia et Avitellina........

animaux

4 animaux

6 animaux

\section{Nematodes et Cestodes :}

- Oesophagostomum et Moniezia.

- Oesophagostomum et Stilesia .

- Oesophagostomum et Avitellina

- Oesophagostomum, Moniezia et Stilesia .................

- Oesophagostomum, Moniezia et Avitellina

2 animaux

16 animaux

18 animaux

3 animaux

- Oesophagostomum, Moniezia, Stilesia et Avitellina ........

- Oesophagostomum, Stilesia et Avitellina ................

- Oesophagostomum, Haemonchus et Moniezia .............

- Oesophagostomum Haemonchus, Stilesia et Avitellina ........

- Oesophagostomum, Haemonchus, Moniezia, Stilesia et Avitellina

- Haemonchus et Moniezia ....

- Haemonchus, Stilesia et Avitellina ...................

- Haemonchus, Moniezia, Stilesia et Avitellina ...........

10 animaux

11 animaux

30 animaux

2 animaux

17 animaux

6 animaux

2 animaux

3 animaux

1 animal

$\mathrm{Au}$ total 81 p. 100 des animaux révèlent à l'autopsie des associations parasitaires Nématodes-Cestodes plus ou moins étendues. 
Il nous a paru intéressant, connaissant l'action séparée du dithiocarbamate de pipérazine (9) et celle de l'arséniate de plomb $(10)$, de rechercher ce que pourrait donner le mélange de ces deux anthelminthiques dans la lutte contre les associations parasitaires dont nous venons de parler.

\section{I. - TECHNIQUE ET MÉTHODE}

\section{A. Les anthelminthiques :}

$1^{0}$ Le Dithiocarbamate de piperazine. Le produit que nous avons utilisé $\left({ }^{a}\right)$ se présente sous la forme d'une poudre jaune granuleuse, stable, pratiquement insoluble dans l'eau. Ce corps renferme de la pipérazine et du sulfure de carbone qui se libère sous l'action du suc gastrique contenu dans la caillette. On connaît les propriétés anthelminthiques très intéressantes du sulfure de carbone sur les nématodes des dernières portions du tube digestif. D'après Gordon (7), il serait aussi très actif sur Haemonchus contortus.

Le dithiocarbamate de pipérazine français, ou carbodithioate, est semblable à un autre dérivé de la pipérazine fabriqué en Angleterre $\left({ }^{b}\right)$ et qui est formé également de sulfure de carbone libérable et de pipérazine (11).

C'est Leiper le premier en 1954 (15) qui recherche l'action du "Safersan " sur Ascaris lumbricoides et Oesophagostomum dentatum du porc. Gordon (6), en Australie, dans une note succincte donne une première vue d'ensemble de l'activité de ce produit sur Oesophagostomum columbianum, Oesophagostomum venulosum, Haemonchus contortus, Chabertia ovina, Trichostrongylus spp. du mouton. Fin 1955, sont entrepris au laboratoire de Farcha (Tchad), des travaux sur l'action de la "Choisine " sur Oesophagostomum columbianum et Haemonchus contortus des ovins (9). Début 1957, Gordon publie les résultats définitifs de ses essais avec le "Safersan " (7).

Les résultats de toutes ces expériences montrent que les produits à base de pipérazinesulfure de carbone présentent le plus grand intérêt dans la lutte contre Oesophagostomum columbianum et Haemonchus contortus du mouton.

$2^{\circ}$ L'Arséniate de plomb. Ce produit est largement utilisé en France depuis plus de 10 ans (Professeur Guilhon) contre Moniezia expansa et son action a fait l'objet d'une mise au point

(a) $(6086$ R.P.) Choisine Spécia.

(b) Safersan, Boots pure drug Co. LTD. récente (3). Il est en outre actif contre a'autres Anoplocéphalinae (10) tels que Stilesia globipunctata, Avitellina centripunctata et Moniezia benedeni.

L'arséniate de plomb que nous avons employé ( $\left.{ }^{c}\right)$ se présente sous la forme d'une poudre dense, de couleur blanchâtre tirant sur le violet; insoluble dans l'eau. Ce corps est vendu également : soit sous forme d'une poudre blanche $\left({ }^{d}\right)$ dont l'activité est rigoureusement la même que celle du "Salvator 98 " soit sous forme de comprimés ( $\left.{ }^{(}\right)$où l'arséniate se trouve associé à d'autres sels minéraux agis sant comme excitants du péristaltisme intestinal.

\section{B. Doses et modes d'administration :}

L'arséniate de plomb a été administré à la dose de $1 \mathrm{~g}$ par animal et le dithiocarbamate de pipérazine à la dose de $125 \mathrm{mg}$ par kilogramme de poids vif; les deux corps versés dans une bouteille sont mélangés intimement.

Une certaine quantité d'eau (un demi-litre environ) est ensuite ajoutée au mélange. Il est recommandé d'agiter vigoureusement le produit obtenu, les deux anthelminthiques insolubles ayant tendance à tomber au fond du récipient. Aucun phénomène de décomposition n'a été observé.

II n'est pas nécessaire de mettre préalablement l'animal à la diète ni de faire suivre le traitement d'une purgation quelconque.

\section{Animaux d'expérience :}

Trente-cing moutons ont subi le traitement précédent à savoir : un lot de 10 femelles pleines, un lot de 6 jeunes de moins de $20 \mathrm{~kg}$ et deux lots de 19 adultes de 25 à $40 \mathrm{~kg}$. Tous ces lots provenaient des mêmes régions et les animaux, à peu près homogènes en matière $d$ 'infestation parasitaire, ont été placés dans les mêmes conditions.

En outre, 31 témoins ont été utilisés.

\section{Les tests :}

Les animaux ont été soumis à trois séries de tests :

10 Des examens coprologiques pour les nématodes (Haemonchus contortus et Oesophagostomum columbianum). Nous avons calculé la moyenne du nombre d'cufs émis dans chaque

(c) Salvator 98 .

(d) Arséniate de plomb à usage vétérinaire des laboratoires Procida.

(e) Moniecidine des laboratoires Thersia. 
lot 6 jours avant et 6 jours après le traitement. La comparaison de ces deux séries de chiffres nous donne le pourcentage de réduction. La même opération a été effectuée dans les lots témoins.

$2^{\circ}$ Dans un second temps, nous avons recherché la moyenne du nombre de parasites (ou de poids pour les cestodes) présents à l'autopsie. Par comparaison avec le même chiffre moyen pris sur les animaux témoins de chaque lot, nous obtenons le pourcentage d'efficacité deś deux corps associés pour le lot considéré.

$3^{\circ}$ Les résultats concernant chaque animal et chaque type de parasite figurent aux tableaux annexes : III, IV, V.

II. - RESULTATS. Tableaux I et II (ci-après)

TABLEAU I : OEUFS (NEMATODES)

\begin{tabular}{|c|c|c|c|c|c|c|c|c|c|c|c|c|}
\hline & \multicolumn{3}{|c|}{$\begin{array}{c}\text { Lot } 1: \text { q pleines } \\
10 \text { traitées } \\
6 \text { témoins. }\end{array}$} & \multicolumn{3}{|c|}{$\begin{array}{l}\text { Lot } 2 \text { : Jeunes } \\
6 \text { traités } \\
6 \text { témoing }\end{array}$} & \multicolumn{3}{|c|}{$\begin{array}{l}\text { Lot } 3 \text { : Adultes } \\
13 \text { traités } \\
13 \text { témoins }\end{array}$} & \multicolumn{3}{|c|}{$\begin{array}{c}\text { I.ot } 4 \text { : Adultes } \\
6 \text { traités } \\
6 \text { témoins }\end{array}$} \\
\hline & Av.tr. & Ap.tr. & \% réd. & Av.tr. & Ap.tr. & \% réd. & Av.tr. & Ap.tr. & \% réd. & Av.tr. & Ap.tr. & \% réd. \\
\hline $\begin{array}{c}\text { Moyenne des oeufs } \\
\text { émis par les } \\
\text { animaux soumis } \\
\text { à l'expérience }\end{array}$ & 46 & 19 & 59 & 16 & 11 & 31,3 & 76 & 52 & 31,6 & 252 & 249 & 1,3 \\
\hline $\begin{array}{c}\text { Moyenne des oeufs } \\
\text { émis par les } \\
\text { ténoins }\end{array}$ & 37 & 30 & 19 & 87 & 126 & 0 & 188 & 170 & 10 & 249 & 416 & 0 \\
\hline
\end{tabular}

$\Delta v_{\text {.tr. }}=$ avant traitement

Ap.tr. = apris traitenent

\% réd. = pourcentage de réduction

TABLEAU II - PARASITES TROUVES A L'AUTOPSIR (en grammes)

\begin{tabular}{|c|c|c|c|c|}
\hline Lots & Parasites & $\begin{array}{c}\text { Parasites reetant chez } \\
\text { les animaux traités } \\
\text { (moyenne) }\end{array}$ & $\begin{array}{c}\text { Parasites restant chez } \\
\text { les témoins } \\
\text { (moyenne) }\end{array}$ & $\begin{array}{l}\text { Pourcentage } \\
\text { d'efficacité }\end{array}$ \\
\hline $\begin{array}{cc}\text { Lot } & 1 \\
1 & 2 \\
1 & 3 \\
" & 4\end{array}$ & $\begin{array}{c}\text { Oesophagostomum } \\
\text { columbianum }\end{array}$ & $\begin{array}{l}0,4 \\
0 \\
0 \\
1,1\end{array}$ & $\begin{array}{c}3 \\
12 \\
8,5 \\
21\end{array}$ & $\begin{array}{l}86,7 \\
100 \\
100 \\
94,8\end{array}$ \\
\hline $\begin{array}{cc}\text { Lot } & 1 \\
11 & 2 \\
\text { I" } & 3\end{array}$ & $\begin{array}{l}\text { Heemonchus } \\
\text { contortus }\end{array}$ & $\begin{array}{l}0 \\
0,3 \\
23\end{array}$ & $\begin{array}{l}0,16 \\
5,9 \\
98\end{array}$ & $\begin{array}{l}100 \\
95 \\
76,6\end{array}$ \\
\hline $\begin{array}{cc}\text { Lot } & 1 \\
" & 2 \\
" & 3 \\
" & 4\end{array}$ & $\begin{array}{l}\text { Moniezia expansa } \\
\text { et } \\
\text { Moniezia benedeni }\end{array}$ & $\begin{array}{l}0 \\
0 \\
0 \\
0\end{array}$ & $\begin{array}{l}1,5 \\
3,5 \\
2 \\
10\end{array}$ & $\begin{array}{l}100 \\
100 \\
100 \\
100\end{array}$ \\
\hline $\begin{array}{cc}\text { Lot } & 1 \\
11 & 2 \\
" & 3 \\
" & 4\end{array}$ & $\begin{array}{l}\text { Stilesig } \\
\text { globipunctata }\end{array}$ & $\begin{array}{l}0,4 \\
0 \\
0 \\
0,16\end{array}$ & $\begin{array}{l}3,4 \\
1,6 \\
3,5 \\
2,6\end{array}$ & $\begin{array}{l}83,3 \\
100 \\
100 \\
93,4\end{array}$ \\
\hline $\begin{array}{cc}\text { Lot } & 1 \\
" 1 & 2 \\
" & 3 \\
" & 4\end{array}$ & $\begin{array}{c}\text { Aritellina } \\
\text { centripunctata }\end{array}$ & $\begin{array}{l}0 \\
0 \\
0 \\
0\end{array}$ & $\begin{array}{l}0,75 \\
0,6 \\
7,2 \\
30\end{array}$ & $\begin{array}{l}100 \\
100 \\
100 \\
100\end{array}$ \\
\hline
\end{tabular}




\section{III. - DISCUSSION}

1. Lé pourcentage global de réduction des cufs de nématodes après administration du mélange, varie de 2,3 à 59 p. 100 selon les lots. Cette faible diminution tient à plusieurs causes : existence chez certains animaux traités de quelques Haemonchus et Oesophagostomum non détruits; ponte accélérée des femelles après déparasitage, ce qui augmente la moyenne du nombre d'oufs émis ; destruction dans le tube digestif des femelles mûres d'Haemonchus avec libération massive d'oufs, les Oesophagostomum étant en général retrouvés intacts à l'extérieur.

Individuellement, entre le quatrième et le sixième jour faisant suite au traitement, on assiste à la disparition progressive des cufs de nématodes dans les cxcréments sauf chez les animaux qui hébergent encore des Haemonchus ou des Oesophagostomum adultes.

2. L'efficacité réelle du mélange dithiocarbamate + arséniate de plomb se traduit de la façon suivante :

a) Sur Moniezia expansa, Moniezia benedeni et Avitellina centripunctata, destruction totale des cestodes dans tous les cas.

b) Sur Stilesia globipunctata, destruction totale chez 33 animaux; deux autres présentaient encore des Stilesia adultes en faible quantité. Ces résultats ne concordent pas exactement avec ceux enregistrés précédemment (10) où le pourcentage d'efficacité sur Stilesia globipunctata était de 100 p. 100 dans tous les cas. Il semble que la différence enregistrée puisse s'expliquer ainsi : les Stilesia sont des Anoplocephalinae fortement accrochés par leurs ventouses à la muqueuse du duodénum où ils déterminent la formation de nodules plus ou moins gros. $\mathrm{Au}$ contraire, les Avitellina et les Moniezia adhèrent à peine à la muqueuse; ils seront détruits très vite et très facilement par l'arséniate de plomb. De par leur mode de fixation, les Stilesia paraissent déjà à priori bien plus difficiles à tuer et à détacher que les précédents parasites.

En outre, les expériences précédentes (10) avaient porté sur des moutons pesant de 23 à $35 \mathrm{~kg}$, chacun ayant reçu un gramme d'arséniate de plomb. Dans le cas présent, nous avons affaire chez les femelles pleines et dans le deuxième lot d'adultes, à quelques ovins pesant plus de $35 \mathrm{~kg}$ (38 à $42 \mathrm{~kg}$ ). Ce sont ceux-là quí n’ont pu être débarrassés de leurs Stilesia.

La conclusion est alors facile à tirer : pour les animaux lourds, au-delà de $35 \mathrm{~kg}$, la dose préconisée jusquà maintenant est insuffisante en ce qui concerne les Stilesia et on a intérêt à augmenter quelque peu la dose d'arséniate de plomb. Quelques essais ultérieurs (non encore publiés) permettent de penser que la dose de 1,2 $\mathrm{g}$ d'arséniate de plomb par tête est efficace sur tous les types de cestodes rencontrés sur les ovins de ces régions.

c) Sur Haemonchus contortus, l'action de la Choisine varie considérablement d'un lot à un autre et d'un animal à l'autre : 100 p. 100 d'efficacité chez les jeunes, 95 p. 100 et 76 p. 100 chez les adultes, résultats qui confirment ce qui a été dit précédemment (9). Plus le nombre d'Haemonchus augmente, plus le pourcentage d'efficacité de la Choisine diminue; plus le poids des animaux est faible, plus l'activité de l'anthelminthique est grande. Nous verrons au paragraphe suivant ce qu'il faut en penser.

d) Sur Oesophagostomum columbianum, il y a disparition complète des nématodes, avec évacuation dans les excréments chez 33 animaux (sur 35) ; chez les deux autres, la destruction n'est que partielle (95 p. 100 et 76 p. 100).

D'après Gordon (7), citant Leiper (15), une grande partie de l'activité dú "Safersan" sur Haemonchus contortus et Oesophagostomum columbianum est liée à la quantité de sulfure de carbone produit au contact des sucs gastriques. Chez les moutons jeunes ou de poids moyen, traités à la dose de $125 \mathrm{mg}$ par $\mathrm{kg}$ de poids vif, la libération de sulfure de carbone paraît suffisante pour détruire une grosse partie des Haemonchus et la totalité des Oesophagostomum. Par contre, chez les moutons de type lourd $(38 \mathrm{~kg}$ et au-delà), avec la même dose d'anthelminthique, la quantité de sulfure de carbone émis semble insuffisante pour obtenir 100 p. 100 d'efficacité. La dose de $125 \mathrm{mg} / \mathrm{kg}$ est donc une dose limite inférieure.

En outre, avec une telle dose, la difficulté dans ces pays d'apprécier exactement le poids d'un mouton risque de conduire à des erreurs de posologie, à l'administration de quantités de "Choisine " nettement inférieures à celles qui seraient nécessaires et finalement à des résultats peu en rapport avec l'efficacité réelle du produit. C'est ainsi qu'à la bergerie de N'Gouri, sur 95 ovins traités à la dose de $125 \mathrm{mg} / \mathrm{kg}$ et dont les poids avaient été fixés de façon approximative, le pourcentage d'efficacité totale sur Oesophagostomum colum bianum n'a atteint que 85 p. 100 du lot. 
TABIEAU III - RESULTATS INDIVIDUEIS : FEMEIIES PLEINES

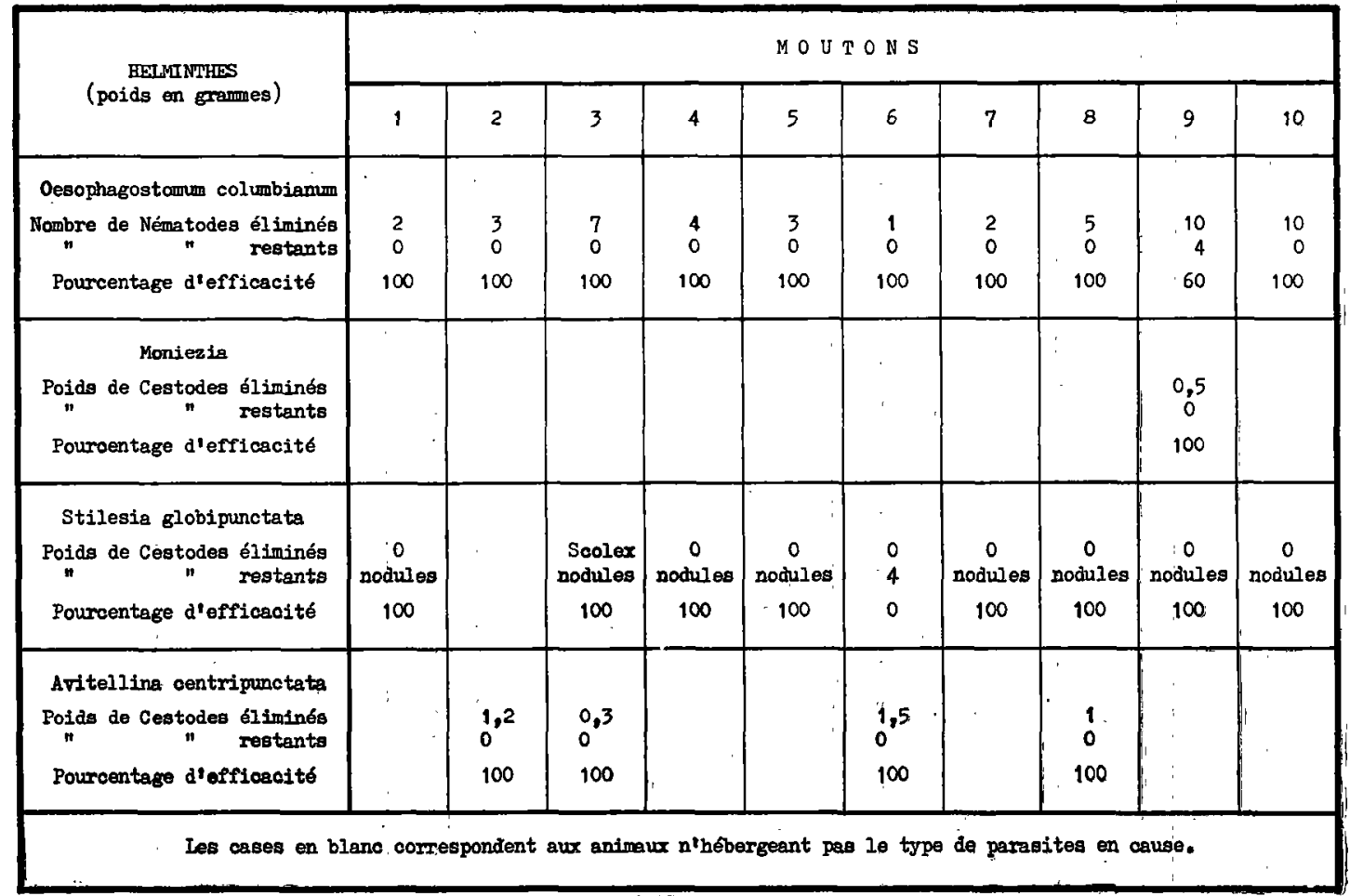

TABIBAU IV : RESULTATS INDIVIDUELS : JEUNES

\begin{tabular}{|c|c|c|c|c|c|c|}
\hline & & & $J E \cup \mathbb{N} E S$ & $M O U T O H S$ & . & \\
\hline . & 1 & 2 & 3 & 4 & 5 & 6 \\
\hline $\begin{array}{l}\text { Oesophagostomum columbianum } \\
\text { Nombre d'helminthes óliminéo } \\
\text { " " restants } \\
\text { Pourcentage d'efficaoité }\end{array}$ & $\begin{array}{r}1 \\
0 \\
100\end{array}$ & $\begin{array}{r}3 \\
0 \\
100\end{array}$ & $\begin{array}{r}9 \\
0 \\
100\end{array}$ & $\begin{array}{r}7 \\
0 \\
\quad 100\end{array}$ & $\begin{array}{r}13 \\
0 \\
100\end{array}$ & $\begin{array}{r}1 \\
0 \\
100\end{array}$ \\
\hline $\begin{array}{l}\text { Haemonchus contortus } \\
\text { Nambre d'helminthes éliminés } \\
\text { " " reotants } \\
\text { Pourcentage d'efficacité }\end{array}$ & & . & - & . & . & $\begin{array}{r}1 \\
0 \\
100\end{array}$ \\
\hline 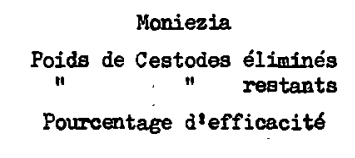 & $\begin{array}{c}1,5 \\
0 \\
100\end{array}$ & $\begin{array}{c}3,5 \\
0 \\
100\end{array}$ & $\begin{array}{r}7 \\
0 \\
100\end{array}$ & . & $\begin{array}{c}0,5 \\
0 \\
100\end{array}$ & $\begin{array}{r}3 \\
0 \\
100\end{array}$ \\
\hline $\begin{array}{l}\text { Stilesia clobipunctata } \\
\text { Poids de Cestodes éliminés } \\
\text { " restants } \\
\text { Pourcentage d'efficacité }\end{array}$ & $\begin{array}{c}0 \\
\text { nodules } \\
100\end{array}$ & $\begin{array}{c}0 \\
\text { nocturies } \\
100\end{array}$ & $\begin{array}{c}\begin{array}{c}0 \\
\text { nodules }\end{array} \\
100\end{array}$ & & $\begin{array}{c}0 \\
\text { nodules } \\
100\end{array}$ & $\begin{array}{c}0 \\
\text { nodules } \\
100\end{array}$ \\
\hline $\begin{array}{l}\text { Avitellina centripunctata } \\
\text { Poids de cestodes éliminés } \\
\text { " restants } \\
\text { Pourcentage defficacité }\end{array}$ & $\begin{array}{c}0,5 \\
0 \\
100\end{array}$ & & $\begin{array}{r}3 \\
0 \\
100\end{array}$ & $\begin{array}{c}0,6 \\
0 \\
100\end{array}$ & & $\begin{array}{c}2,5 \\
0 \\
100\end{array}$ \\
\hline
\end{tabular}




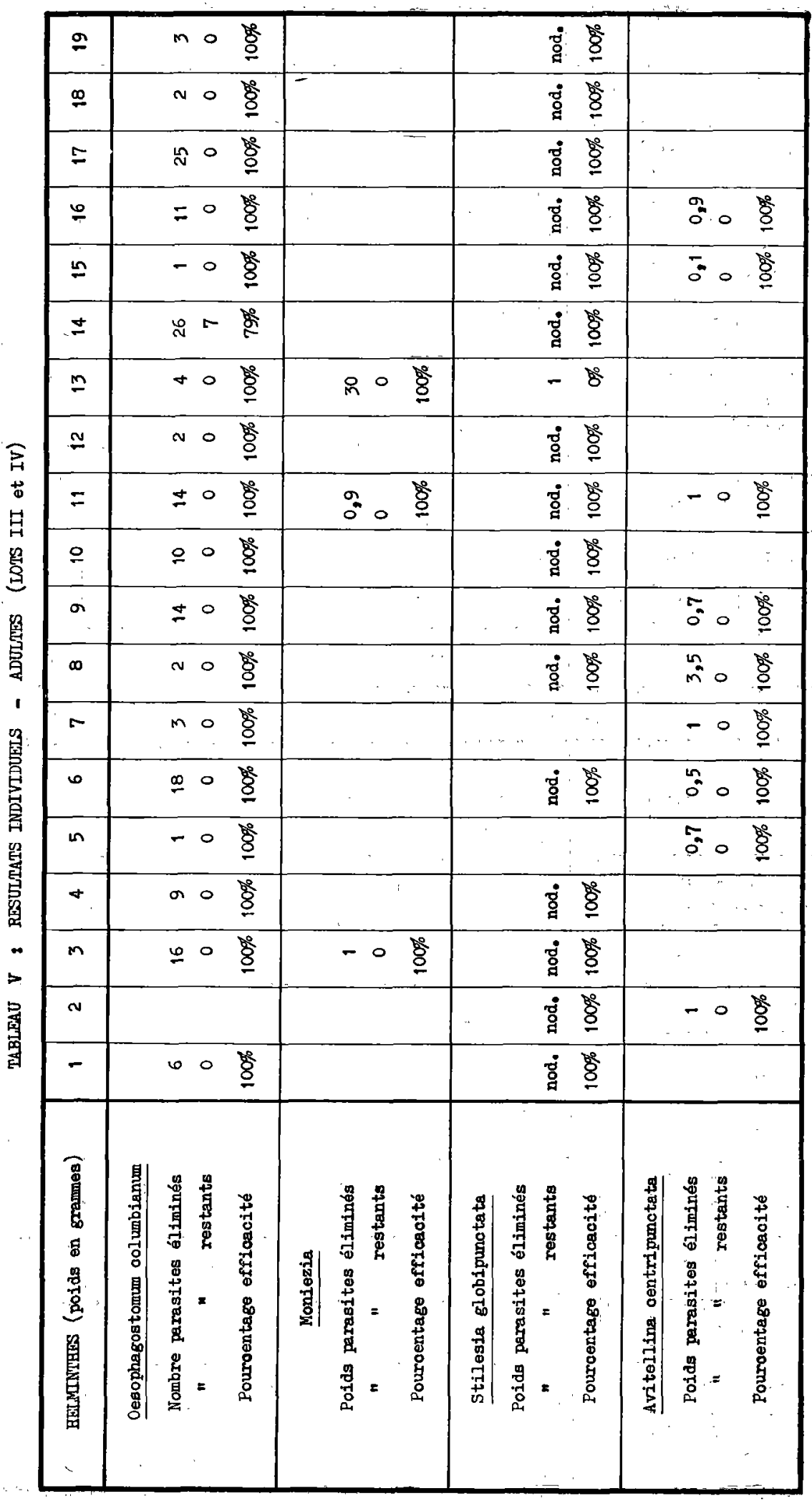


Dans ces conditions, il est apparu nécessaire d'augmenter la dose préconisée (9) et de la porter aux environs de $140-145 \mathrm{mg} / \mathrm{kg}$. Il s'agit là d'une dose très large, tenant compte du poids élevé de certains moutons, des erreurs de posologie et des pertes inévitables avec un produit insoluble quand on le distribue au pistolet.

3. Le mélange a été parfaitement toléré tant chez les adultes que chez les jeunes femelles pleines.

\section{IV. - MODE D'ACTION DU MÉLANGE}

10 Les deux corps associés ont une action anthelminthique rapide.

L'évacuation débute, pour tous les parasites 24 heures après le traitement ; elle est au maximum 48 heures après ; elle est pratiquement terminée dans les 72 heures.

Aucun des parasites rencontrés dans les excréments immédiatement après leur expulsion n'était encore vivant.

EVACUATION TOTALE CHEZ 35 OVINS

\begin{tabular}{|c|c|c|c|c|}
\hline HETLINTHES & $24 \mathrm{~h}$ & $36 \mathrm{~h}$ & $48 \mathrm{~h}$ & $72 \mathrm{~h}$ \\
\hline $\begin{array}{l}\text { Oesophagostomum } \\
\text { columbianum }\end{array}$ & 5 ovins & 5 ovins & 23 oving & 2 ovins \\
\hline Moniezia & 1 ovin & 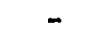 & 5 ovins & 3 ovins \\
\hline Avitelling & 3 ovins & 2 ovins & 10 oving & 3 oving \\
\hline Stileaia & - & - & 1 ovin & - \\
\hline
\end{tabular}

$2^{\circ} \mathrm{Du}$ point du vue action propre des anthelminthiques, Gordon (7) émet l'hypothèse que le dithiocarbamate pourrait être absorbé dans les premières voies de l'appareil digestif, lancé dans la circulation et éliminé au niveau du gros intestin où il agirait sur Oesophagostomum columbianum.

\section{V. - TOXICITÉ}

La toxicité de l'arséniate de plomb pour les moutons du Tchad est connue (10) : la dose mortelle est voisine de 4 grammes par animal.

Par contre, on ne sait encore que peu de chose de la toxicité du dithiocarbamate de pipérazine. Leiper (15) avec le "Safersan " ne réussit pas à intoxiquer des porcs avec $500 \mathrm{mg} / \mathrm{kg}$, ni des volailles avec des doses de 2.640 et $2.500 \mathrm{mg} / \mathrm{kg}$. Chez les moutons avec de la "Choisine", nous avons administré des doses de $400 \mathrm{mg} / \mathrm{kg}$ sans que les animaux en aient été le moins du monde incommodés, si ce n'est un peu de tristesse et d'inappétence dans les heures qui ont suivi l'administration du produit.

Le mélange arséniate + dithiocarbamate se révèle mortel pour le mouton lorsque l'on atteint la dose toxique de l'arséniate de plomb (4 grammes).

\section{VI. - CONCLUSIONS}

1. Les moutons de la zone sahélienne du Tchad hébergent de nombreux parasites dont les plus importants sont Moniezia expansa et Moniezia benedeni, Stilesia globipunctata, Avitellina centripunctata, Oesophagostomum columbianum et Haemonchus contortus. Ces Helminthes se trouvent associés dans 80 p. 100 des cas environ.

2. Dans le but de détruire ces associations parasitaires, l'action du mélange arséniate de plomb (1 g par tête) + dithiocarbamate de pipérazine (125 mg par $\mathrm{kg}$ de poids vif) a été essayée sur 35 moutons comprenant des adultes, des femelles pleines et des jeunes.

3. Le mélange des deux corps ne donne heu à aucun phénomène de décomposition et s'administre parfaitement bien à la bouteille, à condition d'agiter vigoureusement, les deux corps étant insolubles. $\mathrm{Ni}$ la diète préalable, ni la purgation après le traitement ne se révèlent nécessaires.

Le mélange est parfaitement toléré par tous les animaux.

4. L'efficacité du produit est totale sur Moniezia expansa, Moniezia benedeni et Avitellina centripunctata.

5. Sur Haemonchus contortus les résultats sont variables d'un lot à l'autre.

6. Sur Oesophagostomum columbianum, deux animaux présentaient encore quelques parasites à l'autopsie. La non destruction de ces nématodes parait en relation, chez les animaux d'un poids élevé, à une libération insuffisante du sulfure de carbone, produit par le dithiocarbamate au contact des sucs gastriques de la caillette.

7. Sur Stilesia globipunctata, les résultats 
sont les mêmes que précédemment. Là encore, la dose préconisée est trop faible chez les animaux dépassant 35 kilogrammes.

8. Les doses de $1 \mathrm{~g}$ d'arséniate de plomb par tête et de $125 \mathrm{mg}$ de dithiocarbamate par kilogramme de poids vif sont donc dés doses minima. Dans la pratique, il est conseillé de préférence, en tenant compte de tous les aléas d'un traitement antiparasitaire, d'employer l'arséniate à la dose de $1,2 \mathrm{~g}$ par animal et de dithiocarbamate à la dose de 140 à $145 \mathrm{mg}$ par kilogramme de poids vif.

9. Les parasites sont tous totalement évacués dans les 72 heures qui suivent l'administration du mélange.

L'évacuation maximum a lieu 48 heures après le traitement.

10. La toxicité du mélange est celle de l'arséniate de plomb.

(Section d'Helminthologie Laboratoire de Farcha Fort-Lamy (T chad).)

\section{BIBLIOGRAPHIE}

1. Allen (R.:W.) et Jongeling (G.-H.). The Efficacy of Lead Arsenate in Removing Moniezia, from Lambs. N. Amer. Vet., 1948, 29, 645 .

2. Anonymous.. - Treatment Removal of Tapeworm. Mississipi Farm Res., 1946, 9. 3.

3. EuzeBy (J.). - Le Teniasis des ruminants et son traitement. Rev. Med. Vét., 1957, 20, 178-84.

4. Foster (A.-O.) et Haberman (R.-T.). Lead Arsenate for Removal of Ruminants Tapeworms. J. Amer. Vet. Med. Ass., 1948, 113, 51.3.

5. Foster (A.-O.). - Critical Review of Present Day Treatments of Parasitic Infections, Giving List of Drugs. Inter. Vet. Congress (15 th), Stockholm. Proceedings, Part I, Vol. 1, 458-68.

6. GoRdon (H.-Mcl.). - Anthelminthic Effects of Piperazine on Helminths of Sheep. Austr. Vet. J., 1955, 31, 52.
7. Gordon (H.-Mcl.). - Studies on Anthel minthics for Sheep. Austr. Vet. J., 1957, 33; $1-7$.

8. Graber (M.) et Receveur (P.). - Parasitisme interne du mouton en zone sahélienne. Oesophagostomose nodulaire en particulier. Rev. Elev. Méd. vét. Pays trop., 1956, 9, 5-20.

9. Graber (M.). - Action d'un nouvel anthelminthique : le dithiocarbamate de pipérazine sur les nématodes du mouton. Rev. Elev. 'Méd. vét. Pays trop., 1956, 9, 141-7.

10. Graber (M.). - Action de l'arséniate de plomb sur divers anoplocephalidae du mouton. Rev. Elev. Méd. vét. Pays trop., 1947, 10, 119-28.

11. Gretillat (S.). - Essai de traitement des helminthiases intestinale et pulmo. naire du porcelet à Madagascar. Rev. Elev. Méd. vét. Pays trop., 1957, 10, 5-14.

12. Haberman (R.-T.) and Carlson (F.-L.). Lead Arsenate Relieves Scouring in Lambs Due to Tapeworms Infestation. vét. Méd., 1946, 41, 306-10.

13. Harwood (P.-D.). - The Use of Lead Arsenate Mixed With Phenothiazine for the Removal of Tapeworms from Sheeps and Goats. Proc. Helm. Soc. Washington, 1953, 20, 29-31.

14. Hutchins (D.-R.). - Lead Poisoning in Cattle. Austr. Vet. J. 1955, 12, 317-9.

15. Leiper (J.-W.-G.). - The Piperazine Compound $v 19$ for the Removal of Ascaris and Oesophagostomum from the Pig. Vet. Rec. 1954, 66, 596-9.

16. Mc Mulloch (E.-C.) and ST John (J.-L.). - Lead Arsenate Poisoning in Sheep and Cattle. J. Amer. Vet. Med. Ass., 1940, 98, 321-6.

17. Mc Culloch (E.-C.) and Mc Cloy (J.-E.). - Treatment of Ovine Teniasis with Lead Arsenate. J. Amer. Vet. Med. Ass., 1941, 99, 496-7. 
18. Morgan (B.), Pope (A.) andSorensen (D.-E.) - The Efficacy of Lead Arsenate for the Common Tapeworm of Sheep. Vet. Med., 1950, 45, 9, 370-2.

19. Oliver Bill. - Effective Treatment for Tapeworms in Sheep, Goats and Cattle. Sheep and Goat Raiser, 1945, 25, 545 .

20. RADELEFF (R.-D.). - Lead Arsenate an Effective Taeniacide for Domestical Ruminants. Vet. Med., 1944, 39, 453-54.

21. Simms (B.-T.). - Lead Arsenate effective in removing sheep Tapeworms and checking diarrhea in lamb. In Report of Chief of the bureau of animal Industry, 1947, 1946, 17.
22. St John (J.-L.), Mc Mulloch (E.-C.), Sotola (J.) and TodHanter (E.-N.). Toxicity to sheep of Lead Arsenate and Lead Arsenate spray residues. J. Agric. Res, 1940, 80, 317-29.

23. Ward (J.-W.) and Scales (J.-W.). Studies made of Lead Arsenate for sheep Tapeworm. Farm. Res., 1946, $9,6$.

24. Ward (J.-W.) and Scales (J.-W.). - A preliminary report on the use of Lead Arsenate for removal of sheep Tapeworm. J. Amer. Vet. Med. Ass., 1946, 103, 425-26.

25. WhitTen (L.-K.). - The treatment of Tapeworm infestations in man and animals. Vet. Rev. Ann., 1956, 2, $1,1,24$.

\section{SUMMARY}

\section{The association of piperazine dithiocarbaimate and lead arsenate as an anthelmintic for sheep}

In the Sahalien zone of Tchad, sheep harbour numerous species of cestodes and nematodes of which the following principal species may be found in assocation in 80 per cent of cases : Moniezia expansa and Moniezia benedeni, Stilesia globipunctata, Avitellina centripunctata, Esophagostomum columbianum and Haemonchus contortus.

An anthelmintic mixture of piperazine dithiocarbamate $(125 \mathrm{mg} / \mathrm{kg})$ and lead arsenate $(1 \mathrm{~g}$ per head) is efficacious in 92 per cent of animals.

Large sized animals at this dosage retain a proportion of the Stilesia and $F_{\text {sophagostomum }}$ burden. The above dosages are regarded, therefore, as minimal and for complete efficacity should be increased to $140-145 \mathrm{mg} / \mathrm{kg}$ and $1.2 \mathrm{~g}$ per head respectively.

\section{RESUMEN}

La asociación dithiocarbamato de piperazina.arseniato de plomo en la lucha contra diversos helmintos (céstodos y nemátodos) del carnero.

En la zona de Sahel en Tchad, los carneros albergan numerosos céstodos y nemátodos cuyos principales son : Moniezia expansa y Moniezia benedeni, Stilesia globipunctata, Avitellina centripunctata, Oesophagostomum columbianum y Haemonchus contortus que se encuentran asociados en alrededor del 80 por 100 de los casos.

La mezela dithiocarbamato de piperazina $(125 \mathrm{mg} / \mathrm{kg})+$ arseniato de plomo ( $1 \mathrm{gm}$ por cabeza) es eficaz en el 92 por 100 de los animales. En los carneros de gran talla persisten sinembargo algunos Stilesia globipunctata y algunos Oesophagostomum columbianum. El autor piensa que las dosis preconizadas son dosis minimas y sería mejor utilizar para obtener eficacia total, una mezela compuesta de arseniato de plomo a la dosis de 1,2 gm por cabeza y dithiocarbamato de piperazine a la dosis de 140-145 mg por kilogramo de peso vivo. 